Check for updates

Cite this: RSC Adv., 2018, 8, 37151

Received 2nd October 2018 Accepted 28th October 2018

DOI: $10.1039 / c 8 r a 08156 e$

rsc.li/rsc-advances

\section{Electrospun cellulose acetate doped with astaxanthin derivatives from Haematococcus pluvialis for in vivo anti-aging activity $\dagger$}

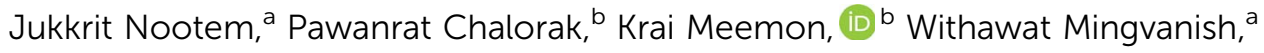 \\ Kornkanya Pratumyot, ${ }^{a}$ Leela Ruckthong, ${ }^{a}$ Choladda Srisuwannaket ${ }^{a}$ \\ and Nakorn Niamnont (D)*a
}

\begin{abstract}
This research aims to study the release, in vivo anti-aging activity against Caenorhabditis elegans and stability of astaxanthins in a crude acetone extract of Haematococcus pluvialis from electrospun cellulose acetate (CA) nanofibers. The content and 2,2-diphenyl-1-picryl-hydrazyl-hydrate (DPPH) radical scavenging activity of astaxanthins in the crude extract were also determined. The content of astaxanthins was reported in terms of total carotenoid content (TCC) and found to be $10.75 \pm 0.16 \mathrm{mg}$ $\mathrm{gcE}^{-1}$. IC 50 of DPPH radical scavenging activity for astaxanthins was $233.33 \pm 4.18 \mu \mathrm{g} \mathrm{mL}^{-1}$. It has been well known that astaxanthins are very unstable under environmental conditions, so the electrospinning technique was used to enhance their stability. In order to fabricate CA nanofibers containing a crude acetone extract of $H$. pluvialis, various solvent systems and percent loading of the crude acetone extract were studied. The optimal solvent system for fabrication of CA nanofibers was the acetone/ dimethylformamide (DMF) system $(2: 1 \mathrm{v} / \mathrm{v})$ with incorporation of $0.25 \% \mathrm{v} / \mathrm{v}$ Tween 80 , resulting in good morphology of CA nanofibers with av. $420 \mathrm{~nm}$ diameter. The loading efficiency (\%) of the crude astaxanthins extract was $5 \% \mathrm{w} / \mathrm{w}$ of $\mathrm{CA}$. With regard to the results of the in vivo oxidative stress assay, $C$. elegans pre-treated with $200 \mu \mathrm{g} \mathrm{mL}^{-1}$ of the crude extract had a survival percent of 56 after administration of $250 \mathrm{mM}$ of paraquat for $8 \mathrm{~h}$. Under phosphate-buffered saline (pH 7.4) containing $10 \%$ $\mathrm{v} / \mathrm{v}$ acetone, the release of astaxanthins from the CA nanofibers loaded with the crude extract exhibited a prolonged profile. The stability of astaxanthins in electrospun CA nanofibers was examined using the freeze-thaw cycle testing through a DPPH radical scavenging assay. It was found that their stability was significantly different $(P<0.05)$ after the $12^{\text {th }}$ freeze-thaw cycle compared with the crude extract.
\end{abstract}

\section{Introduction}

Astaxanthin and its esters are naturally occurring and blood-red carotenoid pigments. They have been found in rainwater microalgae (Haematococcus pluvialis), the yeast fungus called Xanthophyllomyces dendrorhous (also known as Phaffia), Antarctic krill, marine copepods, deep sea shrimp, and edible crab shells. ${ }^{1,2}$ However, astaxanthins has been mainly found in Haematococcus pluvialis. Astaxanthin is considered as a natural super anti-oxidant as compared with the naturally occurring carotenoids. $^{3}$ For examples, astaxanthin exhibited 10 times more antioxidant activity than zeaxanthin, lutein,

\footnotetext{
${ }^{a}$ Organic Synthesis, Electrochemistry \& Natural Product Research Unit, Department of Chemistry, Faculty of Science, King Mongkut's University of Technology Thonburi, Bangkok, 10140, Thailand. E-mail: nakorn.nia@kmutt.ac.th

${ }^{b}$ Department of Anatomy, Faculty of Science, Mahidol University, Bangkok, 10400, Thailand
}

$\uparrow$ Electronic supplementary information (ESI) available. See DOI: 10.1039/c8ra08156e canthaxanthin and $\beta$-carotene, and 100 times higher than vitamin E. ${ }^{4}$ Other benefits of astaxanthin in biological activities are anti-inflammation, anti-diabetic activity, anticancer activity, anti-tyrosinase activity, etc. ${ }^{5,6}$

Most structures of natural astaxanthin have been found in the form of mono- and diesters; $70 \%$ monoesters of astaxanthins, $10 \%$ diesters, $5 \%$ free astaxanthin and the remaining $15 \%$ contains a mixture of $\beta$-carotene, canthaxanthin, lutein and other carotenoids. Because of their structure containing high multiple bonds, they are easily oxidized and unstable upon exposure to heat, light and oxygen, which result in the decrease in their biological activities. ${ }^{7}$ One simple and versatile technique for helping the astaxanthin stability is encapsulation with chitosan, cyclodextrin, calcium alginate, etc. ${ }^{8-10}$ However, the encapsulation technique is limited with difficulty of handling, low loading of bioactive compounds and upscaling for mass production. $^{\mathbf{1 0}}$ A new technique for delivery of bioactive compounds is electrospinning technique, which produced continuous nano to submicro fibers using electrostatic forces. ${ }^{11-13}$ The characteristic advantages of encapsulation 
technique for delivery of bioactive compounds include high drug loading, high encapsulation efficiency, variety of polymers compatible with bioactive compounds, ability to modify drug release rate, process simplicity and cost effectiveness. ${ }^{\mathbf{1 4 , 1 5}}$

Furthermore, fibers produced using electrospinning have high surface area-to-volume and aspect ratios, which can promote the release of bioactive compounds. ${ }^{16,17}$ The most widely used matrix polymer for electrospinning technique is cellulose acetate (CA) due to its biodegradable and non-toxic properties. ${ }^{18}$ Since CA is not too hydrophobic and not hydrophilic, CA enables water to permeate into fibers and make hydrophobic bioactive compounds diffuse to human skin or organism. Many researches have been reported on the study of the release of bioactive compounds such as vitamin E, vitamin $A$, and gallic acid from CA nanofibers. They showed a significant increase in their releasing profile against a media buffer as compared with a casting film. ${ }^{\mathbf{1 9 , 2 0}}$

In this work, we have reported our attempt to enhance the stability of astaxanthins extracted from $H$. pluvialis by doping it with CA fibers upon electrospinning technique. The ability of drug carrier has been tested through in vitro $\mathrm{DPPH}$ radical scavenging activity and in vivo oxidative stress assay with $C$. elegans. These results will facilitate the implementation of electrospinning techniques to stabilize astaxanthins and control quantity of astaxanthins released.

\section{Results and discussion}

\section{Extraction and characterization of astaxanthin derivatives in H. pluvialis}

Astaxanthin derivatives in $H$. pluvialis were extracted with acetone with its percent yield of $21.50 \pm 3.52 \%(\mathrm{w} / \mathrm{w})$. The crude astaxanthin extract was then purified by column chromatography resulting in $4.22 \pm 2.05 \%(\mathrm{w} / \mathrm{w})$ of astaxanthin derivatives and accounted for $0.91 \pm 0.15 \%(\mathrm{w} / \mathrm{w})$ of dry biomass. ${ }^{1} \mathrm{H}-\mathrm{MNR}$ and ${ }^{13} \mathrm{C}$-NMR spectroscopic techniques were used to characterize and identify as astaxanthin esters; mainly astaxanthin monoesters of fatty acids (Fig. S1-S3 in ESI $\dagger$ ). According to the research work of Lorenz et al., ${ }^{21}$ almost $70 \%$ of the astaxanthin derivatives were found to be astaxanthin monoester, while the remaining constituted astaxanthin diester, astaxanthin free, lutein, canthaxanthin and $\beta$-carotene. Since the amount of purified astaxanthin monoester was relatively small. So it was used in the form of the crude extract for further experiments.

\section{Determination of total carotenoid content and DPPH radical scavenging activity of the crude astaxanthin extract from $\boldsymbol{H}$. pluvialis}

The secondary metabolites reported to be found in $H$. pluvialis contain chlorophyll $a$, chlorophyll $b$, and carotenoids and their derivatives. The levels of chlorophyll $a$, chlorophyll $b$ and total carotenoid contents were determined using UV-Vis spectrophotometer (Table S1 $\dagger$ ). The amounts of chlorophyll $b$ and chlorophyll $a$ in the crude extract were $2.54 \mathrm{mg} \mathrm{gcE}^{-1}$, and $8.72 \mathrm{mg} \mathrm{gcE}{ }^{-1}$, respectively. Total carotenoid content was found to be $10.75 \mathrm{mg} \mathrm{gcE}^{-1}$. However, under the proper stress conditions, Haematococcus encysts and produces high concentrations of carotenoids, which facilitates its own protection against light and oxygen. ${ }^{1}$ With regarding to $\mathrm{DPPH}$ radical scavenging testing, this crude extract gave $\mathrm{IC}_{50} 233.33 \pm 4.18(\mu \mathrm{g}$ $\left.\mathrm{mL}^{-1}\right)$.

\section{The effects of solvent systems and Tween 80 on the morphology of CA nanofibers}

The distance between the tip and the corrector on fiber morphology is significant. So, the suitable distance was seated at $15 \mathrm{~cm} .{ }^{22}$ This research aimed to study the effects of solvent systems and surfactant. The morphology of electrospun nanofibers from various types of solvent systems with different ratios was shown in Fig. 1. The average diameters of fibers obtained from the acetone/DMF system with the volume ratios of $2: 1$, $3: 1$ and $4: 1$ were $725 \pm 419,1016 \pm 653$ and $1709 \pm 787 \mathrm{~nm}$, respectively; whereas the relative ratios appeared to be $2538 \pm$ $1654,3006 \pm 1305$ and $3668 \pm 1751 \mathrm{~nm}$ in the EA/DMF system (Fig. 2). Obviously, the different solvent systems dramatically affected the morphology of CA nanofibers due to their different polarity, conductivity, and evaporation rate. ${ }^{\mathbf{1 4}}$ In terms of solvent types, acetone has higher polarity and evaporation rate than EA, thus leading to the different formations of solid-state characteristics of CA nanofibers. At the same ratios of the solvent systems, the average diameters of CA nanofibers were significantly different at $P<0.05$. The acetone/DMF system possessed a proper evaporation rate, subsequently making the CA nanofibers smaller in diameters, while the EA/DMF system was hard to vaporize and revealed non-uniform and larger diameters. ${ }^{23}$ Regardless of solvent systems, an increasing ratio of acetone and EA influenced the morphology of fibers which significantly affected their diameters $(P<0.05)$. Rapid evaporation of solvents caused in the coagulation of solid-state at the needle tip prior to elongation and resulted in a broad diameter distribution. Additionally, a co-solvent system with an acetone/ DMF ratio of 4:1 demonstrated high amount of beads compared to the EA/DMF system at the same ratio. The best characteristic of fibers obtained from the acetone/DMF system at a ratio of $2: 1$ was therefore collected to study the effect of Tween 80 surfactant on its morphology. Since Tween 80 is nonionic surfactant, it could not interrupt with the

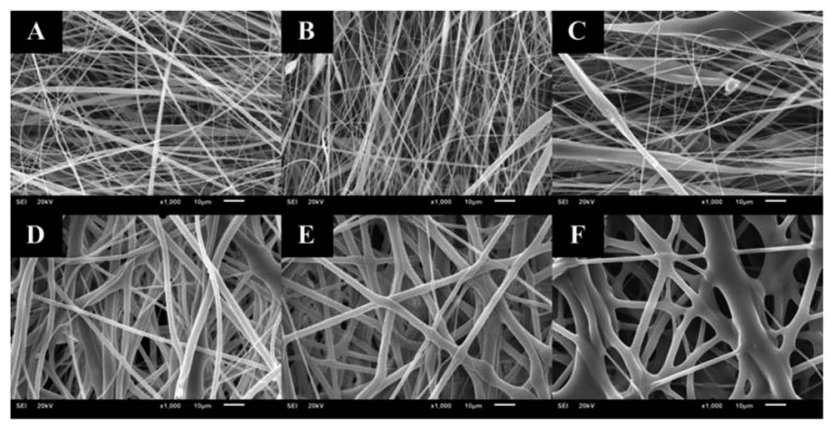

Fig. 1 CA electrospun nanofibers from various types of solvent system and volumetric ratios including: (A) acetone/DMF at $2: 1$, (B) acetone/ DMF at $3: 1,(C)$ acetone/DMF at $4: 1$, (D) EA/DMF at $2: 1$, (E) EA/DMF at $3: 1$, and (F) EA/DMF at $4: 1$, respectively. 


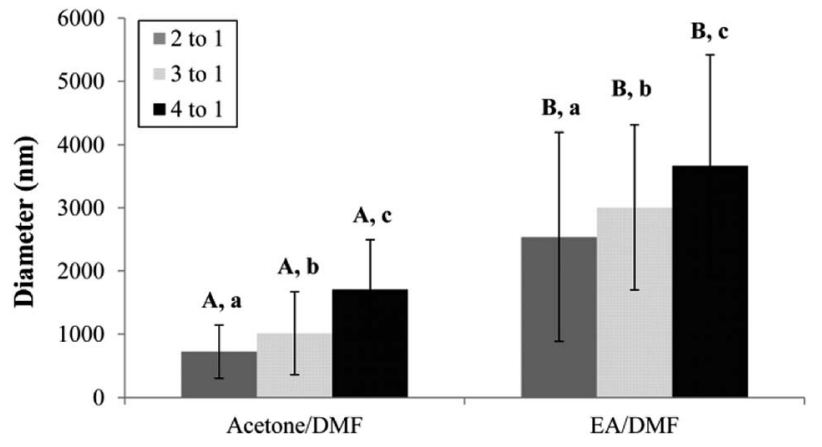

Solvent system

Fig. 2 The average diameter of CA electrospun nanofibers from various types of solvent system and volumetric ratios. ${ }^{\mathrm{A}, \mathrm{B}}$ Means with different capital letter superscripts are significantly different $(P<0.05)$ at the same ratios of solvent.

electrospinning process compared with other cation or anionic surfactants. ${ }^{24}$ Moreover, Tween 80 also exhibits low toxicity and causes less irritation to human skin, which makes it suitable for pharmaceutical and cosmetic applications. ${ }^{25}$ The addition of Tween 80 at $0.25,0.5,1$ and $2 \% \mathrm{v} / \mathrm{v}$ to a solution of CA decreased fibers' average diameter and reduced the standard deviation (Fig. 3 and 4 ) to be $420 \pm 163,419 \pm 198,405 \pm 170$ and $434 \pm$ $203 \mathrm{~nm}$, respectively as compared with the average diameter of the surfactant free CA nanofibers $(725 \pm 419 \mathrm{~nm})$. The average diameter of nanofibers containing Tween 80 was significantly different $(P<0.05)$ from those without Tween 80 incorporation. ${ }^{26}$ When increasing Tween 80 to a proper volume, the surface tension still decreased and the average diameter of fibers became smaller, while excess volume at $4 \% \mathrm{v} / \mathrm{v}$ of Tween 80 resulted in a slight increase in fiber average diameter. Tween 80 is a surfactant with high molecular weight and high level of volatility. It could therefore affect the solvent by increasing the evaporation rate of the polymer solution. The obtained result is consistent with the research work of Jia et al., ${ }^{27}$ in which it was stated that the exceed amount of non-ionic surfactant caused formation of free micelles that consequently disturbs the electrospinning process. As a consequence, relatively larger average diameter of nanofiber was generated. However, the average diameters of CA nanofibers obtained from $4 \% \mathrm{v} / \mathrm{v}$ Tween 80 addition appeared to be not significantly different from those

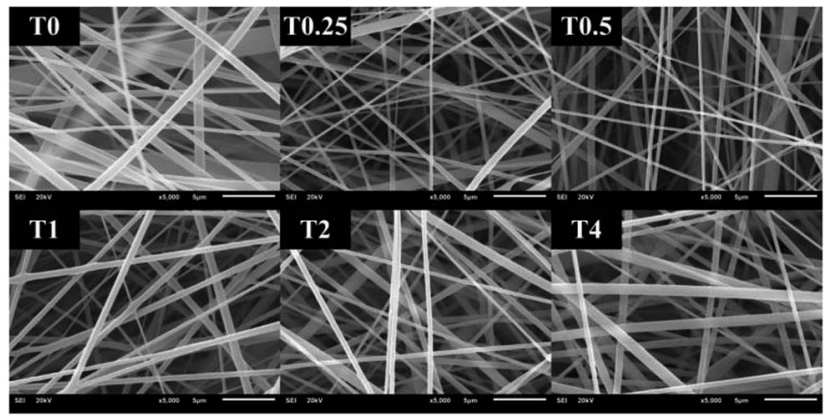

Fig. 3 CA electrospun nanofibers from various volumes of Tween80 including: (TO) 0\%, (TO.25) 0.25\%, (T0.5) 0.5\%, (T1) 1\%, (T2) 2\%, and (T4) $4 \% \mathrm{v} / \mathrm{v}$, respectively.

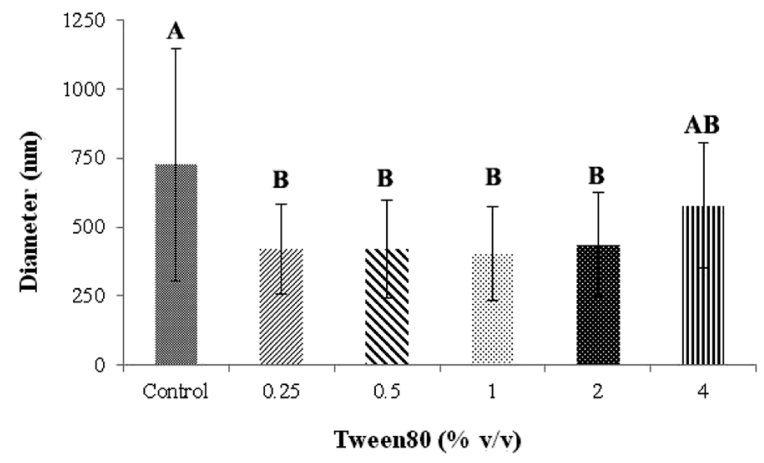

Fig. 4 The average diameter of CA electrospun nanofibers from various volume of Tween80. A,B Means with different capital letter superscripts are significantly different $(P<0.05)$.

obtained from the other volume ratios (0.25-2\%). Because of no significant difference in nanofiber's average diameters among the variation in Tween 80 content, the lowest amount of Tween 80 at $0.25 \% \mathrm{v} / \mathrm{v}$ was collected to study in subsequent processes.

\section{Percent loading (actual drug content)}

Crude astaxanthin extract-loaded CA nanofiber mats were used to investigate the actual drug content after electrospinning procedure. Optimally, the crude astaxanthin extract was dissolved with $17 \% \mathrm{w} / \mathrm{v}$ of CA in acetone/DMF (2:1 ratio) containing $0.25 \% \mathrm{v} / \mathrm{v}$ of Tween 80 to give good morphology of fibers. The crude astaxanthin was obtained in $76.79 \pm 4.98 \%$ (compared to the initial amount of crude astaxanthin in the polymer solution). The loss of drug content during electrospinning was caused from high electrical voltage, oxygen, and heat accumulation at the spinning collector. With all these factors altogether, some of the unsaturated backbone of carotenoid compounds can be oxidized. FT-IR spectra of astaxanthin derivatives (Fig. 5 and Table S2 $\dagger$ ) illustrated a specific peak at $1652 \mathrm{~cm}^{-1}$, which corresponds to a $\mathrm{C}=\mathrm{C}$ bond. Moreover, there is an additional peak at around $1660 \mathrm{~cm}^{-1}$ which is the characteristics of astaxanthin derivatives-loaded CA nanofibers. This showed that the unsaturated $\mathrm{C}=\mathrm{C}$ bond of carotenoid compounds remained stable after fabricating by electrospinning method.

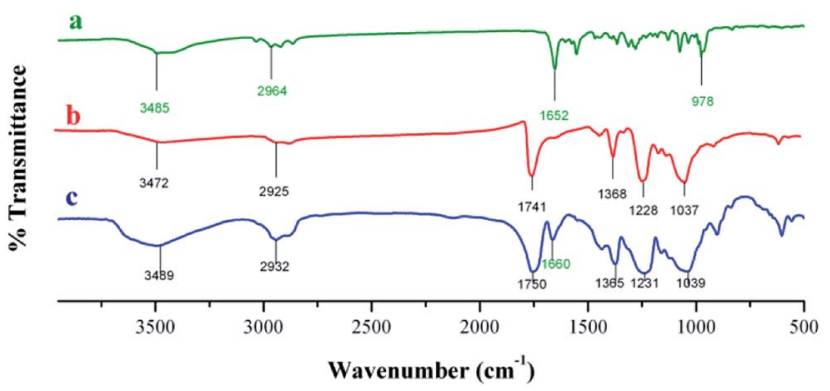

Fig. 5 IR spectra of (a) crude astaxanthin extract, (b) CA electrospun nanofibers, and (c) crude astaxanthin extract loaded CA electrospun nanofibers. 


\section{Effect of percent loading on anti-oxidant activity}

The effect of percent loading of astaxanthins in CA nanofiber fabricated from $17 \% \mathrm{w} / \mathrm{v}$ CA in acetone/DMF (2:1 ratio) and $0.25 \% \mathrm{v} / \mathrm{v}$ of Tween80 was studied by following the DPPH radical scavenging activity. Fig. 6 is the plot of percent loading of astaxanthins against DPPH scavenging rate. The percent loading at 1, 2.5, 5, 7.5 and $10 \% \mathrm{w} / \mathrm{w}$ yielded the DPPH scavenging rates of $54.2 \pm 4.81,73.5 \pm 5.84,110 \pm 7.72,117 \pm 5.63$ and $119 \pm 7.25 \% \mathrm{w} / \mathrm{w}$, respectively. However, the DPPH scavenging rates at the percent loading of $5,7.5$ and $10 \% \mathrm{w} / \mathrm{w}$ were not significantly different $(P<0.05)$. Thus, the lowest percent loading with the highest DPPH scavenging rate of $5 \% \mathrm{w} / \mathrm{w}$ was selected to study in further experiments to save cost of studies and prevent the waste of excess amount of astaxanthins.

\section{Crude astaxanthin renders $C$. elegans resistant to oxidative} stress

To evaluate the astaxanthins' capacity against oxidative stress of C. elegans, the oxidative stress assay was performed under three conditions: pre-treatment with the crude astaxanthin extract, co-treatment with both the crude astaxanthin extract and $250 \mathrm{mM}$ paraquat, and all-treatment. Compared to the oxidative stress resistance of the control group the pre-treated worms with $200 \mu \mathrm{g} \mathrm{mL}^{-1}$ of the crude astaxanthin extract had the significantly increasing survival percentage after being induced with $250 \mathrm{mM}$ paraquat. It was found that the survival percentages were $11.78 \%, 18.21 \%, 22.45 \%, 56.17 \%, 42.30 \%$ and $13.88 \%$ at $2,4,6,8$ and $12 \mathrm{~h}$, respectively, $(p<0.05)$. However, the pre-treatment with 50 and $100 \mu \mathrm{g} \mathrm{mL}^{-1}$ of the crude astaxanthin extract did not significantly increase the survivability. The controlled worms that were only treated with various concentrations of the crude astaxanthin extract could survive for $12 \mathrm{~h}$, which indicates the non-toxicity of the crude astaxanthin extract (Fig. 7a).

\section{Release of astaxanthins-loaded CA nanofibers}

The astaxanthins releasing profile of crude astaxanthin extractloaded CA nanofibers mat is presented in Fig. 8. Increment of time resulted in an increase of astaxanthin release, which exhibited three distinct characteristics. Initially, a burst

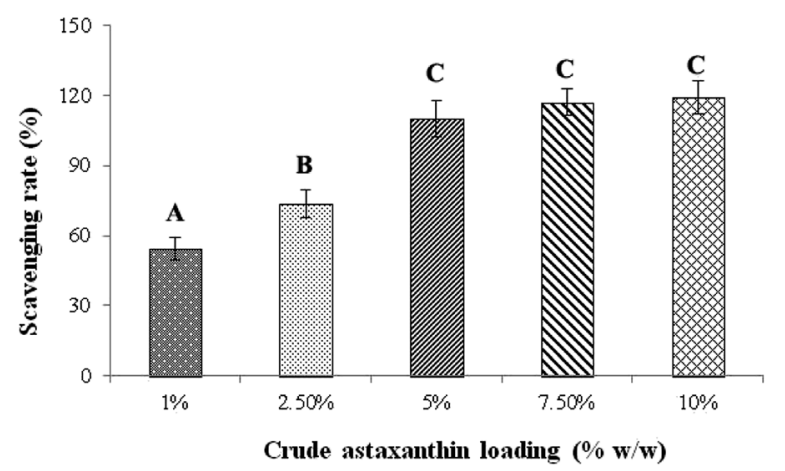

Fig. 6 Effect of percentage loading of crude astaxanthin extract on scavenging rates. $A, B, C$ Means with different capital letter superscripts are significantly different $(P<0.05)$.
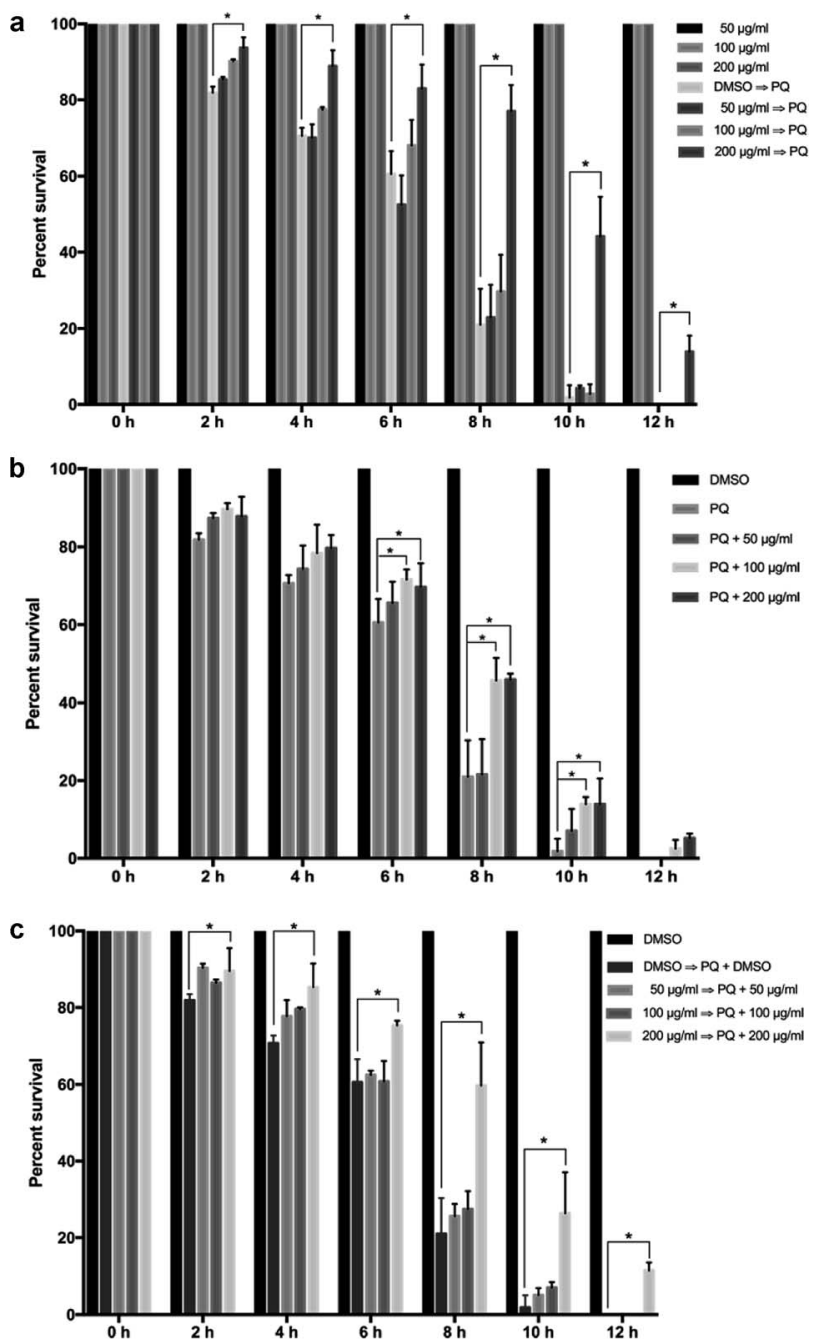

Fig. 7 Oxidative stress resistance of crude astaxanthins at various concentrations in C. elegans for (a) pre-treatment group, (b) cotreatment group, (c) all-treatment group, respectively.

astaxanthins release of $20 \%$ was found within the first $60 \mathrm{~min}$ due to a high surface to volume ratio ${ }^{28}$ of CA-spun fiber that enables the nanofiber mat to swell better in the media. Here, some extent of astaxanthins was emitted into the media and formed micelles with SDS. Moreover, some fatty acids such as

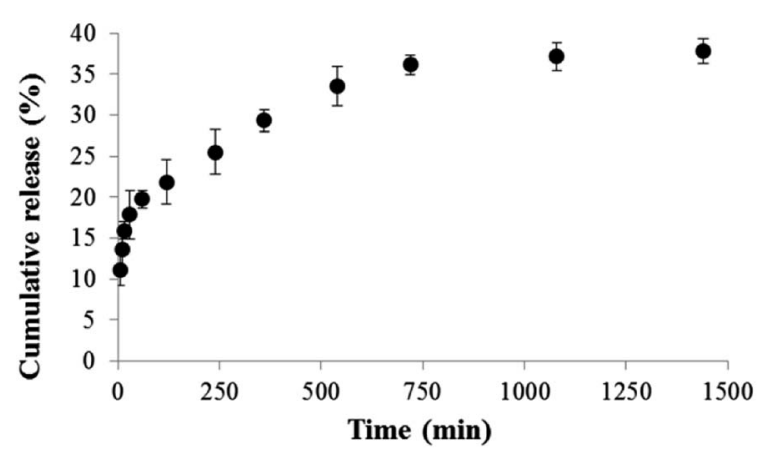

Fig. 8 Cumulative astaxanthins release of crude astaxanthin extract loaded CA electrospun nanofibers in PBS containing $10 \% \mathrm{v} / \mathrm{v}$ of acetone and $0.5 \% \mathrm{w} / \mathrm{v}$ of SDS. 
oleic acid can dissolve well in a media solution containing a small volume of acetone due to their similar polarity. Secondly, in the 60-720 min duration a gradual decrease in astaxanthins releasing rates was due to compound depletion. At this stage, the cumulative astaxanthins release ranged from $\sim 20-35$ in the final interval of the study, the cumulative astaxanthins release was found to be constant. The final cumulative astaxanthins release was $38 \%$ at which the rest of the lipophilic compounds could be entrapped within CA nanofibers. ${ }^{29}$ This can be explained by the fact that the interaction between hydrophobic CA polymeric matrix and hydrophobic astaxanthins was greater than interactions between astaxanthins and water molecules in medium. Tsekova et al., ${ }^{30}$ previously reported that the use of mixed spun-fibers of CA/PVP yielded more efficient release of loaded curcumin than that of CA. This is because PVP is the hydrophilic polymer, so it can completely dissolve in media buffer. As a result curcumin-loaded CA/PVP fibers were released effectively.

\section{Stability of crude astaxanthin extract-loaded CA nanofiber mats}

The stability of astaxanthins-loaded nanofibers was investigated using a freeze-thaw cycle testing. The stability of astaxanthins was determined via their remaining DPPH radical scavenging activity as shown in Fig. 9. At the $0^{\text {th }}-4^{\text {th }}$ cycles, both astaxanthins loaded into CA nanofiber mats and free astaxanthins in crude astaxanthin extract exhibited a rapid fall in DPPH radical scavenging rate upon heating and cooling. A slightly lower DPPH radical scavenging rate of free astaxanthins in crude astaxanthin extract was found at the $8^{\text {th }}$ cycle. The crude astaxanthin extract-loaded CA nanofibers has significantly higher DPPH radical scavenging rate after the $12^{\text {th }}$ cycle as compared with free astaxanthins in crude astaxanthin extract $(P$ $<0.05)$. At the final cycle, it showed an observable difference in their stability with DPPH radical scavenging rate values of 46.18 $\pm 2.21 \%$ for crude astaxanthin extract-loaded CA nanofibers and $26.49 \pm 1.86 \%$ for free astaxanthins in crude astaxanthin extract, respectively. Regarding to the anti-tyrosinase activity, the percentage of tyrosinase inhibition of crude astaxanthin extract-loaded CA nanofibers (35.56 $\pm 3.85 \%)$ was initially

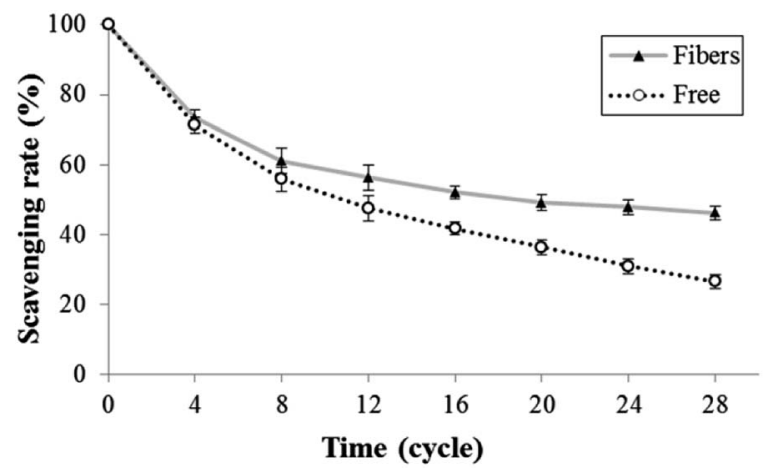

Fig. 9 Antioxidant activity via the freeze-thaw cycle of $(\boldsymbol{\Delta})$ crude astaxanthin extract loaded CA electrospun nanofibers and $(O)$ crude astaxanthin extract. found to be significantly different from that for free astaxanthins in crude astaxanthin extract $(27.22 \pm 2.55 \%)$ at the $4^{\text {th }}$ cycle. The results clearly indicated that CA electrospun nanofibers are a good drug delivery device because it can retain drug stability and at the same time protect the drug from stability testing under simulated conditions (Fig. 10).

\section{Experimental}

\section{Extraction of astaxanthin derivatives from $\mathrm{H}$. pluvialis}

The extraction of astaxanthins from $H$. pluvialis was carried out according to the method of Dong et al. ${ }^{31}$ with slightly modified procedure. Briefly, $10 \mathrm{~g}$ of dried biomass of $H$. pluvialis was disintegrated in $100 \mathrm{~mL}$ of $4 \mathrm{M} \mathrm{HCl}$ with stirring at $500 \mathrm{rpm}$ for $5 \mathrm{~min}$. Then, the sediment was washed by $100 \mathrm{~mL}$ of distilled water twice and extracted with $100 \mathrm{~mL}$ of acetone in ultrasonic bath at $20{ }^{\circ} \mathrm{C}$ for $20 \mathrm{~min}$. The acetone-treated solution was evaporated by rotary evaporator under vacuum condition to obtain a crude astaxanthin extract. The crude extract was kept in a round-bottom flask under nitrogen gas at $4{ }^{\circ} \mathrm{C}$.

\section{Purification and characterization of astaxanthin}

The crude astaxanthin extract was further purified by silica gel column chromatography (60-200 mesh) and eluted with a hexane/EA gradient elution (95:5, $90: 10,85: 15,80: 20$, $75: 25$ and $70: 30 \mathrm{v} / \mathrm{v}$ ). All fractions were traced with thin layer chromatography (TLC). In order to investigate the presence of astaxanthins in the individual fractions, each fraction was characterized by ${ }^{1} \mathrm{H}$ - and ${ }^{13} \mathrm{C}-\mathrm{NMR}$ techniques using Advanced III HD $400 \mathrm{MHz}$ NMR spectrometer (Switzerland) in chloroform-d.

\section{Determination of total carotenoid content in crude astaxanthin extract}

The solutions of the crude astaxanthin extract at the known concentrations $\left(50-200 \mu \mathrm{g} \mathrm{mL}^{-1}\right)$ were prepared in acetone. The determination of total carotenoid content was carried out by UV-Vis spectrophotometer (Hitachi U-2900, Japan) at 470, 645 and $662 \mathrm{~nm}$ following protocols of Lichtenthaler et al. ${ }^{32}$ Total

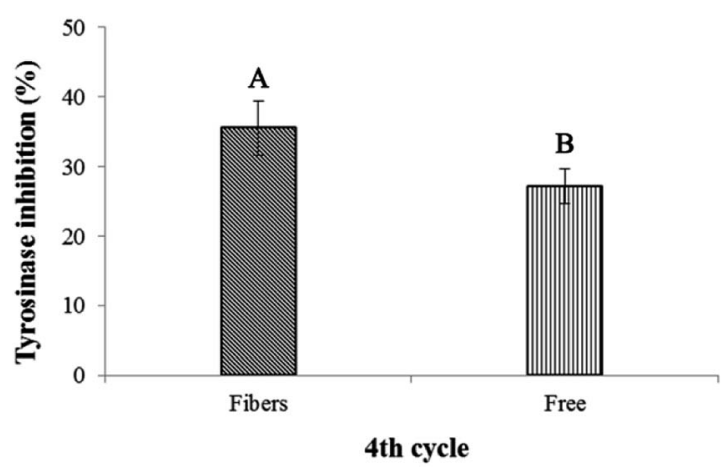

Fig. 10 Tyrosinase inhibitory activity via the freeze-thaw cycle of crude astaxanthin extract loaded CA electrospun nanofibers and crude astaxanthin extract at $4^{\text {th }}$ cycle. ${ }^{A, B}$ Means with different capital letter superscripts are significantly different $(P<0.05)$. 
carotenoid content, chlorophyll $a$ and $b$ were calculated according to the equations below.

$$
\begin{aligned}
& \text { Chlorophyll } a\left(C_{a}, \mathrm{mg} \mathrm{L}^{-1}\right)=11.75 \mathrm{Abs}_{662}-2.35 \mathrm{Abs}_{645} \\
& \text { Chlorophyll } b\left(C_{b}, \mathrm{mg} \mathrm{L}^{-1}\right)=18.61 \mathrm{Abs}_{645}-3.96 \mathrm{Abs}_{662}
\end{aligned}
$$

Total carotenoids $\left(\mathrm{mg} \mathrm{L}^{-1}\right)=\left(1000 \mathrm{Abs}_{470}-2.27 C_{a}-81.4 C_{b}\right) /$ 227

where $\mathrm{Abs}_{470}, \mathrm{Abs}_{645}$ and $\mathrm{Abs}_{662}$ are the absorbance of the solutions at 470, 645 and $662 \mathrm{~nm}$, respectively.

\section{DPPH radical scavenging activity of crude astaxanthin extract}

The anti-oxidant activity of the crude astaxanthin extract was measured according to the method of Kirby and Schmidt ${ }^{33}$ with some modifications. $0.1 \mathrm{mM}$ DPPH solution was prepared in $10 \% \mathrm{v} / \mathrm{v}$ acetone in methanol and sample solutions of predetermined concentrations were also prepared $10 \% \mathrm{v} / \mathrm{v}$ acetone in methanol. Each $100 \mu \mathrm{L}$ of $0.1 \mathrm{mM}$ DPPH solution was added into $100 \mu \mathrm{L}$ of sample solutions. The mixtures were shook and allowed to stand for $30 \mathrm{~min}$ in the dark at room temperature. Then, the absorbance of the mixture was measured by 96 -well plate UV spectrophotometer (Perkin-Elmer; EnSpire Multimode Plate Reader, United States) at $517 \mathrm{~nm}$. The scavenging activity was calculated as following equation.

$$
\text { Scavenging activity }(\%)=\left|\frac{A_{\text {control }}+A_{\text {blank }}-A_{\text {sample }}}{A_{\text {control }}}\right| \times 100
$$

where $A_{\text {control }}, A_{\text {blank }}$ and $A_{\text {sample were the absorbance of } 0.1 \mathrm{mM}}$ DPPH solution in $10 \% \mathrm{v} / \mathrm{v}$ acetone in methanol, the sample solution without $0.1 \mathrm{mM}$ DPPH solution and the sample solution with $0.1 \mathrm{mM}$ DPPH solution, respectively. The scavenging activity value of the crude astaxanthin extract was reported in terms of half maximal inhibitory concentration $\left(\mathrm{IC}_{50}\right)$.

\section{Electrospinning and characterization of CA fiber mats}

Preparation of the CA solutions. $17 \% \mathrm{w} / \mathrm{v}$ CA solutions were prepared in various solvent systems containing either acetone/ DMF or EA/DMF with the volumetric ratios of $2: 1,3: 1$ and $4: 1.17 \% \mathrm{w} / \mathrm{v}$ CA solutions were prepared in various solvent systems containing either acetone/DMF or EA/DMF with the volumetric ratios of $2: 1,3: 1$ and $4: 1$. Then, each polymer solution of known concentration was loaded in a $5 \mathrm{~mL}$ of syringe equipped with a metal needle of an inner diameter of $0.22 \mathrm{~mm}$ with the rate of $2 \mathrm{~mL} \mathrm{~h}^{-1}$.

Electrospinning condition. For electrospinning, the needle was connected to the positive electrode (Gamma high voltage research FL 32174, United States) and the negative electrode was connected to the rotary spinning collector. The needle tip was located at about $15 \mathrm{~cm}$ apart from the collector. Each polymer solution was then injected with applied voltage of 15 $\mathrm{kV}$ and the rotary spinning collector was spun at $120 \mathrm{rpm} \cdot{ }^{34-36}$

Effect of Tween80 on morphology of CA nanofibers. The optimized conditions for electrospinning CA nanofibers were applied for studying the effect of the concentrations of Tween 80 surfactant on its morphology. ${ }^{37}$ Tween 80 surfactant was added into the polymer solution at various concentrations $(0,0.25,0.5$, $1,2$ and $4 \% \mathrm{v} / \mathrm{v})$. In order to examine the morphology of CA nanofiber samples, the samples were dried in a vacuum oven at $50{ }^{\circ} \mathrm{C}$ for $3 \mathrm{~h}$. After that, each sample was cut into small pieces ( 1 $\times 1 \mathrm{~cm})$ and coated with gold. Then, the morphology of CA nanofibers was observed under a scanning electron microscope (JEOL JSM-6610 LV; SEM, United States). The diameter, diameter distribution and uniforming of each CA nanofiber sample was analyzed using Image $1.51 \mathrm{~K}$ software $(n \geq 100) .{ }^{38}$ For each experiment, the average fiber diameter and distribution were determined from 100 measurements of the random nanofibers. All experiments were conducted at room temperature.

\section{Studying of crude astaxanthin extract-loaded CA nanofibers}

Percent loading. The crude astaxanthin extract of a predetermined weight was dissolved in a CA solution of known concentration without adding Tween80 and was electrospun under optimum conditions to afford a CA nanofiber mat. $10 \mathrm{mg}$ of a CA nanofiber mat loaded with the crude astaxanthin extract were re-dissolved in $10 \mathrm{~mL}$ of a mixture of acetone and $N, N$ dimethylacetamide $(2: 1 \mathrm{v} / \mathrm{v})$. Its solution was examined for the amount of astaxanthins using UV-Vis spectrometer at $475 \mathrm{~nm}$ and was reported as mean value $+\mathrm{SD}(n=3)$.

FT-IR analysis. For qualitative analysis, the CA nanofiber mat loaded with the crude astaxanthin extract $(2 \mathrm{~cm} \times 1 \mathrm{~cm})$ was characterized using FT-IR spectrometry (Thermofisher scientific; Nicolet iS5, USA) and its absorption spectra was compared with that without the crude astaxanthin extract. The scanning ranges of spectra were $500-4000 \mathrm{~cm}^{-1}$ with a resolution of $16 \mathrm{~cm}^{-1}$. Each spectrum was recorded for 32 scans.

Effect of percent loading on anti-oxidant activity. In each experiment, a CA solution containing various concentrations of the crude astaxanthin extract $(1 \%, 2.5 \%, 5 \%, 7.5 \%$ and $10 \% \mathrm{w} / \mathrm{w}$ of CA powder) was homogenized for $5 \mathrm{~min}$ in sonication machine and was examined for the DPPH radical scavenging activity. Briefly, $50 \mathrm{mg}$ of each fiber sample was extracted with $5 \mathrm{~mL}$ of $10 \% \mathrm{v} / \mathrm{v}$ of acetone in methanol. Next, the anti-oxidant activity was analysed as mentioned above. ${ }^{31}$

In vivo anti-aging activity by studying the oxidative stress in C. elegans. The maximum effectiveness of percent loading of the crude astaxanthin extract onto CA fibers was used to evaluate the oxidative stress effect in C. elegans $50 \mathrm{mg}$ of the CA nanofibers containing the crude astaxanthin extract was extracted by $5 \mathrm{~mL}$ of ethanol. The evaporated crude extract was dissolved in 1\% DMSO to afford feeding solutions at any concentration that will henceforth explain. C. elegans were handled according to the standard methods described previously. ${ }^{39}$ Large populations of C. elegans were cultured on solid Nematode Growth Media (NGM) agar plate and fed with Escherichia coli OP50 at $20^{\circ} \mathrm{C}$. Synchronized eggs were isolated from gravid adult worms by bleaching solution (12\% NaClO and $10 \% 1 \mathrm{M} \mathrm{NaOH}$ ) and then incubated at $20^{\circ} \mathrm{C}$ overnight to obtain newly hatched L1 larvae. Oxidative stress in C. elegans was induced by exposure of $250 \mathrm{mM}$ paraquat. Synchronized L1 larvae were allowed to develop and grow on agar plates containing OP50 mixed with only 1\% (v/v) DMSO alone (control) or 
the crude extract solutions in 1\% (v/v) DMSO. Fifty worms of $C$. elegans were transferred to 96-well plate containing S-basal medium mixed with OP50 for each concentration of the crude extract. For pre-treatment group, the crude astaxanthin extractpretreated worms were transferred into the wells containing $1 \%$ DMSO or $250 \mathrm{mM}$ paraquat. For co-treatment group, the 1\% (v/ v) DMSO-treated worms were transferred into the wells containing either the mixture of $1 \%(\mathrm{v} / \mathrm{v})$ DMSO and $250 \mathrm{mM}$ paraquat or the crude astaxanthin extract combined with $250 \mathrm{mM}$ paraquat. For all-treatment conditions, the crude astaxanthin extract-pretreated worms were transferred into the wells containing the crude astaxanthin extract alone or crude astaxanthin extract combined with $250 \mathrm{mM}$ paraquat. The worms were incubated at $20{ }^{\circ} \mathrm{C}$ for $12 \mathrm{~h}$. The numbers of live and dead worms were counted and recorded every $2 \mathrm{~h}$. The percent survival was then calculated.

Releasing study. In order to prepare the releasing media of astaxanthin, the releasing media contained $10 \% \mathrm{v} / \mathrm{v}$ of acetone and $0.5 \% \mathrm{w} / \mathrm{v}$ of SDS in PBS solution $\mathrm{pH} 7.4$ to improve their solubility as stated in Taepaiboon et al. ${ }^{19}$ with slightly modified conditions. The crude astaxanthin extract-loaded CA nanofibers were cut to afford a small piece of $50 \mathrm{mg}$ and put in the $50 \mathrm{~mL}$ Erlenmeyer flask containing $20 \mathrm{~mL}$ of releasing media. The flask was covered by aluminium foil and paraffin film and put in a rotary shaker at $200 \mathrm{rpm}$. To investigate the amount of astaxanthin, the absorbance of the samples was measured at $475 \mathrm{~nm}$ at 5, 10, 15, 30, 60, 120, 240, 480, 720 and $1440 \mathrm{~min}$, respectively. The amount of astaxanthins was calculated based on its calibration curve and reported as percentage cumulative release. The fresh media solution was filled at the same volume after sampling.

Stability of crude astaxanthin extract-loaded CA nanofibers. Freeze-thaw cycle method was used to evaluate the stability of astaxanthin derivatives in CA nanofibers compared with that of free crude astaxanthin extract according to $\mathrm{Su}$ et $a{ }^{40}{ }^{\mathbf{4 0}}$ protocol with slight modifications. The accurate concentrations of astaxanthin solution were prepared with and without CA solution. After electrospinning, both of samples were transferred into an amber glass vial and incubated at $-20{ }^{\circ} \mathrm{C}$ for $22 \mathrm{~h}$. After that, frozen-samples were thawed at $50{ }^{\circ} \mathrm{C}$ for $2 \mathrm{~h}$ in an incubator. The freeze-thaw cycle process was repeated for 28 times. The stability of astaxanthins in the crude astaxanthin extract and crude astaxanthin extract loaded CA nanofibers was examined every 4 days using DPPH radical scavenging activity assay. Concisely, $50 \mathrm{mg}$ of nanofibers were extracted with $5 \mathrm{~mL}$ of ethanol. The tyrosinase activity was performed using dopachrome method by Likhitwitayawuid et al. ${ }^{41}$ with some modifications. Mixtures of tyrosinase solution, L-dopa solution, ethanolic samples and phosphate buffer solution were measured for the absorbance at $473 \mathrm{~nm}$ using EnSpire Multimode Plate Reader (Perkin-Elmer, USA). The percentage tyrosinase inhibition was calculated as follows.

$$
\text { Tyrosinase inhibition }(\%)=\left[\frac{(A-B)-(C-D)}{(A-B)}\right] \times 100
$$

where $A$ is the difference of absorbances $\left(\mathrm{Abs}_{20} \min -\mathrm{Abs}_{0 \text { min }}\right)$ of control, $B$ is the difference of absorbances $\left(\mathrm{Abs}_{20 \text { min }}-\mathrm{Abs}_{0 \text { min }}\right)$ of blank of control, $C$ is the difference of absorbances $\left(\mathrm{Abs}_{20} \mathrm{~min}-\right.$ $\left.\mathrm{Abs}_{0 \mathrm{~min}}\right)$ of sample, $D$ is the difference of absorbances $\left(\mathrm{Abs}_{20 \mathrm{~min}}-\right.$ $\mathrm{Abs}_{0 \text { min }}$ ) of blank of sample.

\section{Statistical analysis}

Statistical analyses were carried out by Minitab Statistical Software (Minitab Version 16.2.4.0). All data were demonstrated as mean + SD values and compared to one-way analysis of variance (ANOVA). Tukey's test was used for a determination of the differences. The test with $P<0.05$ was considered as a significant difference. All experiments were performed at least in triplicates.

\section{Conclusion}

The optimal conditions for fabrication of CA electrospun nanofibers were studied. The effects of solvent systems and surfactant on fabrication of CA electrospun nanofibers play an important role in morphology of the fibers. The proper evaporation rate of the solvent systems and the appropriate amount of surfactant exhibited in a smaller average diameter size of fibers with uniform characteristic. The optimized conditions for fabrication of CA electrospun nanofibers was then used to produce crude astaxanthin extract-loaded CA nanofibers. It was found that the released free astaxanthins from crude astaxanthin extract-loaded CA nanofibers exhibited the capacity against the oxidative stress of $C$. elegans with significant values. The releasing profile of fibers in a modified buffer solution was that of a prolonged release. Moreover, the stability of released astaxanthins from the crude astaxanthin extract-loaded CA nanofibers was significantly higher compared to that of free astaxanthins in crude astaxanthin extract under an accelerated condition. The results suggest that drug loaded CA electrospun nanofibers are applicable for future drug delivery control applications.

\section{Conflicts of interest}

There are no conflicts to declare.

\section{Acknowledgements}

The authors gratefully thank the research fund for the Capacity Building Program for New Researcher 2018 from National Research Council of Thailand (NRCT), the scholarship of Faculty of Science, King Mongkut's University of Technology Thonburi and the KMUTT 55th Anniversary Commemorative Fund.

\section{Notes and references}

1 S. Boussiba and A. Vonshak, Plant Cell Physiol., 1991, 32, 1077-1082.

2 EFSA (European Food Safety Authority), EFSA J, 2005, 291, 140.

3 W. Miki, Pure Appl. Chem., 1991, 63, 141-146.

4 Y. M. Naguib, J. Agric. Food Chem., 2000, 48, 1150-1154. 
5 R. R. Ambati, H. N. Sindhuja, S. M. Dharmesh, K. U. Sankar, R. Sarada and G. A. Ravishankar, J. Agric. Food Chem., 2013, 61, 3842-3851.

6 R. R. Ambati, S. M. Phang, S. Ravi and R. G. Aswathanarayana, Mar. Drugs, 2014, 12, 128-152.

7 P. Kidd, Altern. Med. Rev., 2011, 16, 355-365.

8 P. Kittikaiwan, S. Powthongsook, P. Pavasant and A. Shotipruk, Carbohydr. Polym., 2007, 70, 378-385.

9 C. Yuana, L. Dua, Z. Jin and X. Xub, Carbohydr. Polym., 2013, 91, 385-389.

10 S. F. Lin, Y. C. Chen, R. N. Chen, L. C. Chen, H. O. Ho, Y. H. Tsung, M. T. Sheu and D. Z. Liu, PLoS One, 2016, 11, e0153685.

11 P. A. Khan, K. Sasikanth, S. Nama, S. Suresh and B. Brahmaiah, J. Pharm. Innov., 2013, 2, 118-127.

12 H. Li, Y. Xu, H. Xua and J. Chang, J. Mater. Chem. B, 2014, 2, 5492-5510.

13 I. Liakos, L. Rizzello, H. Hajiali, V. Brunetti, R. Carzino, P. P. Pompa, A. Athanassiou and E. Mele, J. Mater. Chem. B, 2015, 3, 1583-1589.

14 J. Wei, J. Lu, Y. Liu, S. Yan and X. Li, J. Mater. Chem. B, 2016, 4, 7155-7167.

15 G. Amariei, K. Boltes, P. Leton, I. Iriepa, I. Moraled and R. Rosal, J. Mater. Chem. B, 2017, 5, 6776-6785.

16 F. A. Anahitai, L. Qun, Y. W. Chan and S. Y. Chan, AAPS PharmSciTech, 2010, 11, 1164-1170.

17 Y. Xiao, M. Wang, L. Lin, L. Du, M. Shen and X. Shi, Mater. Chem. Front., 2018, 2, 891-900.

18 J. Zhang, J. Wu, J. Yu, X. Zhang, J. He and J. Zhang, Mater. Chem. Front., 2017, 1, 1273-1290.

19 P. Taepaiboon, U. Rungsardthong and P. Supaphol, Eur. J. Pharm. Biopharm., 2007, 67, 387-397.

20 M. Phiriyawirut and T. Phaechamud, Open J. Polym. Chem., 2012, 2, 21-29.

21 R. T. Lorenz and G. R. Cysewski, Trends Biotechnol., 2000, 18, 160-167.

22 H. Lee, M. Nishino, D. Sohn, J. S. Lee and I. S. Kim, Cellulose, 2018, 25, 2829-2837.

23 S. Zupancic, S. Baumgartner, Z. Lavrica, M. Petelin and J. Kristl, J. Drug Delivery Sci. Technol., 2015, 30, 408-416.
24 C. Kriege, K. M. Kit, D. J. McClements and J. Weiss, Food Biophys., 2009, 4, 213-228.

25 W. C. Gakenheimer and K. G. Ludwig, Parfuem. Kosmet., 1973, 54, 43.

26 S. Q. Wang, J. H. He and L. Xu, Polym. Int., 2008, 57, 10791082.

27 L. Jia and X. H. Qin, J. Therm. Anal. Calorim., 2013, 112, 595605.

28 A. Talebiana and A. Mansouriana, Mater. Today Commun., 2017, 4, 7065-7069.

29 R. Srikar, A. L. Yarin, C. M. Megaridis, A. V. Bazilevsky and E. Kelley, Langmuir, 2008, 24, 965-974.

30 P. B. Tsekova, M. G. Spasova, N. E. Manolova, N. D. Markova and I. B. Rashkov, Mater. Sci. Eng., C, 2017, 73, 206-214.

31 S. Dong, Y. Huang, R. Zhang, S. Wang and Y. Liu, Sci. World J., 2014, 19, 694305.

32 K. Lichtenthaler and A. R. Welburn, Biochem. Soc. Trans., 1983, 11, 591-592.

33 A. J. Kirby and R. J. Schmidt, J. Ethnopharmacol., 1997, 56, 103-108.

34 B. W. Davis, N. Niamnont, C. D. Hare, M. Sukwattanasinitt and Q. Cheng, ACS Appl. Mater. Interfaces, 2010, 2, 17981803.

35 B. W. Davis, N. Niamnont, R. Dillon, C. J. Bardeen, M. Sukwattanasinitt and Q. Cheng, Langmuir, 2011, 27, 6401-6408.

36 B. W. Davis, A. J. Burris, N. Niamnont, C. D. Hare, C. Y. Chen, M. Sukwattanasinitt and Q. Cheng, Langmuir, 2014, 31, 9616-9622.

37 R. Rosic, P. Kocbek, S. Baumgartner and J. Kristl, J. Drug Delivery Sci. Technol., 2011, 21, 229-236.

38 N. A. Hotaling, K. Bharti, H. Krielc and C. G. Simon, Biomaterials, 2015, 61, 327-338.

39 S. Brenner, Genetics, 1974, 77, 71-94.

40 C. Su, Y. Feng, J. Ye, Y. Zhang, Z. Gao, M. Zhao, N. Yang, K. Nishinaria and Y. Fang, RSC Adv., 2018, 9, 4731-4741.

41 K. Likhitwitayawuid and B. Sritularak, J. Nat. Prod., 2001, 64, 1457-1459. 